\title{
Physics of Atomic-Scale Secondary-Electron Imaging
}

\author{
R.F. Egerton, ${ }^{*}$ D.Su,** J.Wall** and Y. Zhu** \\ * Physics Department, University of Alberta, Edmonton, Canada T6G 2G7 \\ ** Brookhaven National Laboratory, Upton, NY 11973 USA
}

A recent publication [1] presents evidence for secondary-electron images of single atoms on a thin carbon support and of atomic columns in a crystal. Atomic-scale resolution was aided by the use of probe-forming lens corrected for spherical aberration, high-energy $(200 \mathrm{keV})$ incident electrons to reduce chromatic aberration, and a thin sample to minimize the background level due to backscattering. Even so, the achievement of atomic resolution in an SE image has occasioned some surprise.

The surprise is reduced if we accept that, out of the three stages of SE production (generation by inelastic scattering of primary electrons, transport to the surface and emission into vacuum), only the generation process affects spatial resolution. In that case, the main resolution limit is localization of the inelastic scattering, which is described to a good approximation [3] by the Rayleigh criterion:

$$
\mathrm{L} \sim 0.6 \lambda / \sin \alpha
$$

Here $\alpha$ is an average scattering angle that depends on the energy loss $E$ of the primary electrons. Assuming a Lorentzian angular distribution of halfwidth $\theta_{\mathrm{E}}$ and cutoff angle $\theta_{\mathrm{c}}$, the median scattering angle is $\theta_{\mathrm{m}} \sim\left(\theta_{\mathrm{E}} \theta_{\mathrm{c}}\right)^{1 / 2} \sim 0.7\left(\mathrm{E} / \mathrm{E}_{0}\right)^{3 / 4}$. Taking $\mathrm{E}$ as a mean energy loss $\mathrm{E}_{\mathrm{m}} \sim 6.75 \mathrm{Z}$ [2], with $\mathrm{E}_{0}=200 \mathrm{keV}$ and $\alpha=\theta_{\mathrm{m}}$, Eq.(1) gives $\mathrm{L} \sim 0.16 \mathrm{~nm}$ for $\mathrm{Z}=92$. Therefore the observation of single $U$ atoms on thin carbon seems quite plausible. This conclusion is a result of (1) the inverse relationship between $L$ and the width of the angular distribution of scattering, (2) the extended tail of the Lorentzian distribution (giving $\theta_{\mathrm{m}} \gg \theta_{\mathrm{E}}$ ), (3) the large value of $\mathrm{E}_{\mathrm{m}}$ for heavy elements (reflecting substantial inelastic scattering from inner atomic shells) and (4) the belief that the lateral spreading of secondaries that reach the surface has no effect on the spatial resolution.

Even in the case of outer-shell excitation, there appears to be sufficient high-angle scattering to permit atomic resolution, as shown in Fig. 1. The calculation assumes weakly-bound $(13.6 \mathrm{eV})$ electrons and hydrogenic wavefunctions. For energy losses greater than about $60 \mathrm{eV}$, more than half of the stopping power derives from high-angle $(\theta>10 \mathrm{mrad})$ scattering, corresponding to $\mathrm{L}<0.15 \mathrm{~nm}$ according to Eq.(1). This highangle scattering approximates to Rutherford scattering from free electrons, for which the scattering angle is approximately $\left(\mathrm{E} / \mathrm{E}_{0}\right)^{1 / 2} \sim 17 \mathrm{mrad}$ for $\mathrm{E}=60 \mathrm{eV}$ and $\mathrm{E}_{0}=200 \mathrm{keV}$. Since the inelastic scattering from carbon atoms involves predominantly outer-shell electrons, secondary-electron images of graphene appear plausible.

Similar principles may apply to the SE imaging of atomic columns in crystalline specimen. Here the situation is complicated by the Bloch-wave behavior of the primary electrons but there is good reason to believe that the SE signal being imaged is generated 
within a depth of a few nm of the entrance surface, before channeling becomes severe. Because of this low escape depth, the practical problems associated with atomicresolution SE imaging largely revolve around cleanliness of the specimen surface

\section{References}

[1] Y. Zhu et al., Nature Materials 8 (2009) 808.

[2] H. Koppe, Z. Phys. 124 (1948) 658.

[3] R.F. Egerton, Ultramicroscopy 107 (2007) 575.

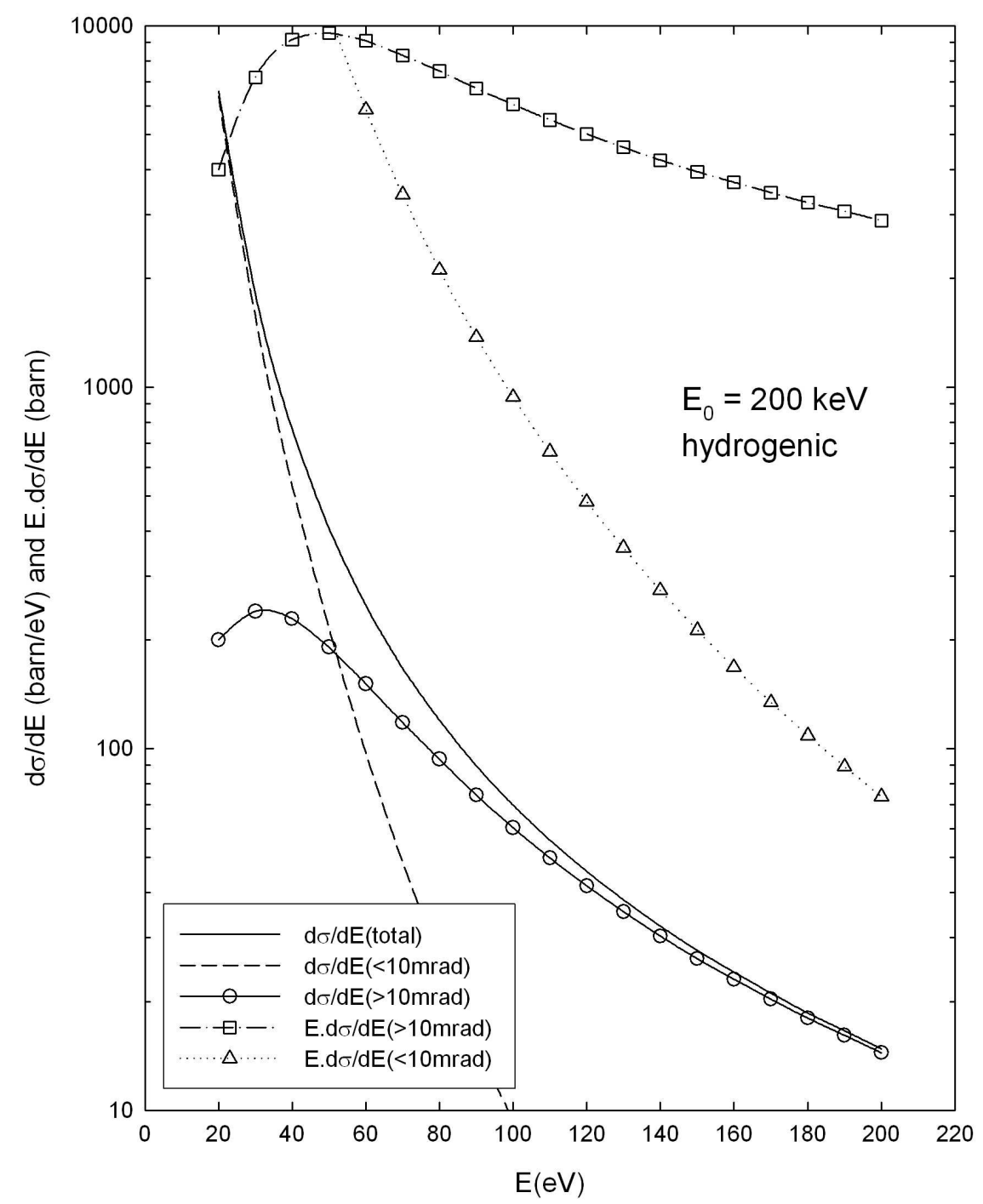

FIG.1: Energy-loss dependence of the differential cross section $\mathrm{d} \sigma / \mathrm{dE}$ for inelastic excitation of a hydrogenic electron by a $200 \mathrm{keV}$ primary electron, summed over all angles of scattering (solid curve), angles up to $10 \mathrm{mrad}$ (dashed curve) and angles greater than $10 \mathrm{mrad}$ (circular data points). Also shown is the stopping power E.d $/ \mathrm{dE}$, representing the energy available to create secondaries from primary electrons that are scattering through angles less than $10 \mathrm{mrad}$ (triangles) and greater than $10 \mathrm{mrad}$ (squares). 